\title{
Identification of transverse spin currents in noncollinear magnetic structures
}

\author{
Jianwei Zhang and Peter M Levy \\ Department of Physics, 4 Washington Place, New York University, New York, New York 10003 \\ Shufeng Zhang \\ Department of Physics and Astronomy, University of Missouri-Columbia, Columbia, Missouri 65211 \\ Vladimir Antropov \\ Ames Research Laboratory, Ames, Iowa 50011
}

(Dated: November 21, 2018; Published date)

\begin{abstract}
We show that the transverse components of spin current in a ferromagnet is linked to an off diagonal spin component of the transmission matrix at interfaces;it has little to do with the mismatch of band structures between dissimilar metals. When we take account of this component,not considered in prior analyses, we find spin torque comes from a region of at lease $3 \mathrm{~nm}$ around an interface.
\end{abstract}

PACS numbers: 72.25.-b, 72.15.Gd, 73.23.-b

Keywords: spin transport,noncollinearity, transverse spin accumulation

Attention has recently been focused on current induced switching of magnetic layers; this idea was proposed in 1996 [1] and has been verified in the past three years. 2] It occurs when one drives an electric current across a multilayered structure in which the magnetic layers are noncollinear. The central idea behind the switching is that when the spin polarized current that develops in a fixed ferromagnetic layer of a multilayered structure impinges on a second noncollinear free magnetic layer the component of the spin current transverse to the magnetization is absorbed. As one assumes the conservation of spin angular momentum this creates the torque on the background magnetization to switch it. One of the unresolved issues is the length scale over which the transverse component of the spin current is absorbed and what material parameters controls it. Here, we point out, that the detail of the transverse spin near the interface does matter in determining the spin transfer torque. The reason is that transport is inherently non-local so that the incident spin polarization at an interface does depend on the detail of the transverse spin accumulation even if this length scale is small, i.e., one must include transverse pin accumulation. Mathematically, the boundary condition solely from the vanishing transverse spin density in ferromagnets is not sufficient to determine the spin torque. If we write the boundary condition to the distribution function on the two sides of an interface, we have found that the truncation of the transmission matrix to a diagonal matrix in spin space misses key relations between the spinor distribution functions on the two sides of an interface, and thus the transport across the entire structure is unable to be self-consistently determined.

We recently found a possible mechanism by which one can inject off diagonal spin distributions into each sheet of the spin split Fermi surface of a magnetic layer, i.e., in the presence a current a spin flip potential can exist at interfaces in noncollinear magnetic multilayers. $[3]$
This additional scattering excites the transverse components of spin currents in magnetic layers that are found by adopting the Boltzmann equation's definition of spin current and then we find there is no discontinuity in the spin current at an interface between two dissimilar layers. This transverse current is different from that evaluated from equilibrium states; [4, [5] they complement one another but the existence of the latter does not negate the former. We evaluate the relevant parameters over which this component is absorbed by using ab-initio band structure calculations and find the characteristic length scale in a typical $3 \mathrm{~d}$ transition-metal ferromagnet $(\mathrm{Co})$ is $3 \mathrm{~nm}$. This is an order of magnitude greater than that found in prior analysis which evaluated a quantum mechanical expectation value of the current over the Fermi surface. [4, 5] As the layers being switched are typically $2-3 \mathrm{~nm}$ this differences is important as it alters one's estimates of the critical current for switching.

The spin current density at a point $r$ can be defined as []

$$
\vec{j}_{\alpha \beta}(r)=\frac{e \hbar}{i m}\left\langle\Psi_{\alpha}^{\dagger}(r) \stackrel{\leftrightarrow}{\nabla}_{r} \Psi_{\beta}(r)\right\rangle
$$

where the expectation value of the operator is taken over a nonequilibrium distribution function and $\alpha, \beta$ are spin indices. This "quantum mechanical" definition of spin current Eq.(1) has been evaluated for a selected set of equilibrium states so as to make a spin polarized current enter a ferromagnet at an angle to the magnetization rather than self-consistently determing the nonequilibrium distribution. [4, 5] Based on this definition the transverse component of the spin current has been shown to be carried by the correlation between the states of opposite spin on the Fermi surface $k_{M}=k_{F \uparrow}, k_{m}=k_{F \downarrow}$. [4] This identification has lead one to conclude that it is the band mismatch between the electronic structures of adjacent layers that causes the polarization of the spin currents to 
change abruptly in a narrow region about an interfaces so that the component of the spin current transverse to the magnetization of a layer is absorbed within about a $\frac{1}{2} \mathrm{~nm}$ of the interface $4,[5]$, i.e., the spin split band structure of the magnetic layers does not allow a spin current transverse to the magnetization to propagate past the interfacial region. 7, 8| We recently showed how in the presence of a current one can inject a spin distribution transverse to the magnetization across an interface with a well defined momentum; [3] here we determine how it propagates in a homogenous layer.

The equation of motion for the electron transport density matrix $\left\langle a_{k \alpha}^{\dagger} a_{k^{\prime} \beta}\right\rangle$, i.e., the quantum or semiclassical Boltzmann equation, in a translationally invariant system and for linear response is an equation for a given momentum state in which states at other momenta enter through the scattering terms. 10] The Wigner transform of these matrix elements, 10 .

$$
f_{\alpha \beta}(k, r)=\frac{e}{(2 \pi \hbar)^{3}} \int d k^{\prime} e^{i k^{\prime} \cdot r}\left\langle a_{k+k^{\prime} / 2, \alpha}^{\dagger} a_{k-k^{\prime} / 2, \beta}\right\rangle
$$

is independent of position $r$ for a system in equilibrium, e.g., the Fermi distribution function. While the perturbation of the electric field in and of itself does not break the translational invariance of a layer the ensuing current crossing an interface does; therefore when a current is driven across a system as a multilayer the out of equilibrium part of $f_{\alpha \beta}(k, r)$ develops a position dependence. In the Boltzmann description the transverse components of the spin current are

$$
\vec{j}_{\alpha \beta}(r)=\frac{1}{(2 \pi \hbar)^{3}} \int d k \vec{v}(k) f_{\alpha \beta}(k, r),
$$

where in linear response $\vec{v}(k)$ is the Fermi velocity of the electron. In principle both definitions Eqs.(11) and (3) give the same current provided sufficient care is taken in their evaluation.

The equation of motion for the density matrices or distribution functions is the Boltzmann equation; here we use the semiclassical version of this equation. In each layer the energy and density vary slowly on the length scale of the Fermi wavelength so that we can limit ourselves to the first term in the gradient expansion of the equation of motion. 11] When we limit ourselves to linear response in the external electric field we find the equations of motion in steady state for the elements of the spinor density matrix for each momentum state on the Fermi surface $k_{p}$ are, [10]

$$
v_{p}^{x} \partial_{x} f_{p}-e E v_{p} \delta\left(\varepsilon-\varepsilon_{F}\right)=-\frac{f_{p}-\left\langle f_{p}\right\rangle}{\tau_{p}}-\frac{f_{p}-\left\langle f_{p^{\prime}}\right\rangle}{\tau_{s f}},
$$

and

$$
v_{p}^{x} \partial_{x} f_{p}^{ \pm} \mp i \frac{J_{p}}{\hbar} f_{p}^{ \pm}=-\frac{f_{p}^{ \pm}-\left\langle f_{p}^{ \pm}\right\rangle}{\tau_{p}},
$$

where we have used a simplified index $p$ to denote the momentum $k_{p}$ of a state on the $n^{t h}$ sheet of the Fermi surface(we suppress this index), $p^{\prime}$ are states of opposite spin to $p$, the average $\left\langle f_{p}\right\rangle$ represents elastic scattering to all states on the Fermi surface, $v_{p}^{x}$ is the component of the Fermi velocity along the electric field $E, J$ is the magnetic part of the energy (see below) and we have limited the current induced variations of the distribution function to those along the growth direction of a multilayered structure $x$ (also the field direction). The diagonal elements $f_{p}=f_{\alpha \alpha}\left(k_{p}, x\right)$ represents the occupancy of the state $k_{p}$; in equilibrium it is given by the Fermi function so that only the spin state $\alpha$ that crosses the Fermi level is occupied while the other is zero and we do not consider it further. The off diagonal elements $f_{p}^{ \pm} \sim f_{\uparrow \downarrow}\left(k_{p}, x\right)$, which we call a current induced spin coherence, $[9]$ represent coherences between the state $k_{p}$ on the Fermi surface and the states with opposite spin; see Eq.(2); these coherences occur when we drive a spin current across a normal metal/ferromagnet (N/F) interface. [3] The scattering terms include those for states of the same spin on the Fermi surface $\tau_{\alpha}^{-1}$ as well as those between sheets of opposite spin $\tau_{s f}^{-1}$; as $\tau_{s f}^{-1} \ll \tau_{\alpha}^{-1}$ we include the latter only to have well defined boundary conditions on our distribution functions.

The solutions for the longitudinal components are well know. 12 From Eqs.(41) and (5) we see that the electric field only creates out of equilibrium longitudinal components of the distribution functions; in a homogeneous magnetic layer there is no coupling to the transverse components. However when the spin current from one layer is injected into another noncollinear magnetic layer the transverse components can be excited. As such they have to be determined by a self consistent calculation of the distribution functions in the different layers of a multilayered structure. When we neglect collisions (the rhs of Eq. (50) the transverse solutions are $f_{p}^{ \pm}(x) \sim \exp \pm \mathbf{i}\left(J_{p} / \hbar v_{p}^{x}\right) x$; when we average this over the Fermi surface we find an interference between individual $p$ or $\mathbf{k}$ states so that the transverse components of the spin currents in the magnetic layers, $j_{x}^{ \pm}(x) \sim \int v_{x}(k) f^{ \pm}(k, x) d k$ (Eq. (3) $)$, can be fit to a form approximating an exponential decay $\sim \exp -x / \lambda_{t r} .13$. In the ballistic regime $\lambda_{t r}=d_{J} \equiv h \overline{v_{F} / J}$ where the bar denotes an average over states on the $n^{\text {th }}$ sheet of the Fermi surface under consideration; 14 while for diffusive systems where we consider the collision terms $\lambda_{t r}=\lambda_{J} \equiv \sqrt{d_{J} \lambda_{m f p} / 3 \pi}$. 115. Therefore when components of the spin current are injected into a magnetic layer that are transverse to its magnetization, we find they propagate a distance $\lambda_{t r}$ before decaying; as this distance is an order of magnitude greater than the Fermi wavelength one can describe the transverse spin currents in the semiclassical Boltzmann approach. As $\lambda_{t r}$ is comparable to the thickness of the magnetic layers undergoing switching one cannot assume the transverse compo- 
nents of spin currents are entirely absorbed in such thin layers.

To evaluate $d_{J}$ we need the Fermi velocity $v_{F}(\mathbf{k})$, and $J(\mathbf{k})$ for each point $\mathbf{k}$ on the $n^{\text {th }}$ sheet of the Fermi surface under consideration; the latter is identified as follows. The energy of a spin polarized band in a uniform ferromagnetic metal can be written as a spinor

$$
\hat{\varepsilon}_{n}(k)=\varepsilon_{n}(k) \mathbf{1}+\frac{1}{2} J_{n}(k) \vec{M} \cdot \vec{\sigma}
$$

where $n$ is the band index, $\vec{M}$ is a unit vector in the direction of the magnetization, and $\vec{\sigma}$ represents the Pauli spin matrices. As such $J_{n}(k)$ is the magnetic part of the band energy. For a band crossing the Fermi surface it is found by identifying the band of opposite spin that is split from the common energy $\varepsilon_{n}(k)$ by $J_{n}(k)$. This requires one to do two band structure calculations, one nonmagnetic in which each band is spin degenerate and another for the fully spin polarized state in which the two spin split states originating from the common degenerate band can be easily identified along directions of high symmetry. 16] With this identification we find

$$
J_{n}(k)=\left(\varepsilon_{n \uparrow}(k)-\varepsilon_{n \downarrow}(k)\right),
$$

where $\varepsilon_{n \sigma}(k)$ are the energies of the spin split band and $n$ is the index for the band that crosses the Fermi surface whose magnetic component we are evaluating. Therefore $J_{n}(k)$ can be found from looking at the band structure of a ferromagnetic metal; it is the difference in energy at constant $k$ between two split bands. While these two bands mostly lie on top of (next to) one another, there are regions of the Brillouin zone where one has to do some further analysis in order to identify the bands which had a common origin in the nonmagnetic phase.

From the band structure for $f c c$ Co we find $d_{J}$ for $\Delta_{1}$ majority states along the $\Gamma-X(100)$ direction is $7.4 \mathrm{~nm}$ but it decreases rapidly off this axis, along $\Gamma-K(110) d_{J}$ is $2.93 \mathrm{~nm}$, along $\Gamma-L(111) d_{J}$ is $1.1 \mathrm{~nm}$, and the average $d_{J}$ for the majority band over the Fermi surface is about $3 \mathrm{~nm}$. 17] Of the three minority spin bands crossing the Fermi surface the one that is the partner of the majority, $\# 3$, has a $d_{J}$ of $0.8 \mathrm{~nm}$ while the other two have even shorter $d_{J}$. Central to the semiclassical description of the transverse spin currents is the condition that they can propagate over distances large compared to the Fermi wavelength; we find that this condition is well satisfied for the $3 \mathrm{~d}$ transition metal ferromagnets for the majority band.

To identify the source of the discontinuity in a spin current at interfaces we have solved the Boltzmann equations Eqs.(4) and (5) for the current across two magnetic layers; while there is an intervening nonmagnetic layer we adopt the usual assumption that its thickness is much less than the spin diffusion length so the spin current is constant across it. For the electronic structure we take a free electron-like model in which the degenerate spin bands are split by the internal fields; the effect of the exchange splitting of the bands is to produce two separate channels of conduction on the Fermi surface, one majority one minority. 18] To keep track of current induced coherences the conduction in each channel is described by a $2 \times 2$ density matrix so that one has to match two $2 \times 2$ density matrices at interfaces to obtain a description of the spin current across a noncollinear multilayered structure, i.e., each spin channel is represented by a spinor distribution function. To perform a self consistent calculation of the distribution functions across a multilayered structure we have to match them at the interfaces by using transmission and reflection coefficients. In addition to the spin diagonal coefficients $T_{m m} \Longrightarrow s s^{\prime}\left(s^{\prime}=s\right)$ previously considered we add the off diagonal coefficient with $s^{\prime} \neq s$ that transmits spin coherence across an N/F interface; [3] therefore we must consider in our matching not only states on the Fermi surface but also those with which electrons form current induced coherences in steady state, i.e., in general we have to match $4 \times 4$ rather than $2 \times 2$ distribution functions across interfaces.

When one limits oneself to spin diagonal transmission coefficients at an interface there is no transmission into off diagonal or transverse elements of the density matrix, i.e., the $f_{\uparrow \downarrow}$, are zero (not excited) in the magnetic layers; as they have been identified as the transverse components of the spin current one arrives at the conclusion that the transverse component of the spin current has been absorbed at the interface. [4, 7] However, when we include the off diagonal transmission coefficient the discontinuity of the transverse component of the spin current at interfaces is removed. In other words without $T_{m m} \Longrightarrow s s^{\prime}$ with $s^{\prime} \neq s$ we find the spin current in a ferromagnetic layer is parallel to the background magnetization and there is an abrupt rotation of the spin current when the layers are noncollinear; with it the spin current is continuous. While transverse spin currents do not exist when one uses the equilibrium distribution that was selected to evaluate Eq. (11), 4, [5] they do exist when we use Eq.(15) to self-consistently evaluate the transverse component of the spin current Eq. (3) in steady state. 19] When we solve the Boltzmann for the spin current across two noncollinear magnetic layers we find its polarization starts to deviate from the background magnetization a distance $\lambda_{t r}$ on either side of the interface and is continuous across it. 20] While the spin accumulation, and therefore the resistance, depends on the reflection and transmission coefficients due to the specular scattering at the interface, the continuity of the spin current is insensitive to them as long as we include $T_{m m} \Longrightarrow s s^{\prime}$ with $s^{\prime} \neq s$; therefore we conclude that their discontinuity is not an indigenous property of noncollinear magnetic layers. We find it is caused when one suppresses the transverse components of the spin currents in the magnetic layers either by using only diagonal transmission coefficients [7] or by using 
only states at the Fermi surface to evaluate the spin current Eq.(11). 4, 5]

In summary, when the steady state spin current is selfconsistently evaluated using Eq. (3) we keep track of the transverse spin accumulation around the interfaces irrespective of its length scale. We find there is no absorption at the interface, it is continuous across it, and the transverse component decays within $3 \mathrm{~nm}$, at least for Co, irrespective of the reflection and transmission coefficients; albeit these coefficients affect the magnitude of the spin currents and resulting torques. Therefore the discontinuity in spin currents at interfaces is not due to band mismatch; rather we find it arises from omitting the off diagonal components of the transmission coefficients and the ensuing transverse spin accumulation. The difference between our result and those which predict spin torques created within $\frac{1}{2} \mathrm{~nm}$ of an interfaces is important as the thickness of layers undergoing magnetization reversal is in the range of $2-3 \mathrm{~nm}$. When we introduce diffuse scattering in the layers we reduce the decay length for the transverse component of the spin current as we found in the spin diffusion model of current induced magnetic switching, 21 but we do not create discontinuities in the current.

We thank Albert Fert for questioning the validity of the spin diffusion model in this context, Ingrid Mertig for identifying the magnetic component of the spinor band energy from ab-initio calculations and together with Peter Zahn for the band structure of $\mathrm{Co}$ and the Fermi velocity across the various sheets of the Fermi surface, and Asya Shpiro for comparing her results from the spin diffusion model to those presented here. Numerous discussions with Andy Kent, Jerry Percus, Bob Richardson and Tycho Sleator at NYU were very useful especially in developing the relevance of coherences in the context of transverse spin currents. This work done under a grant from the National Science Foundation (Grant DMR 0131883 and DMR 0314456). Ames Laboratory is operated for the U.S.Department of Energy by Iowa State University under Contract No. W-7405-82.

[1] J.C. Slonczewski, J. Mag. Mag. Mater. 159, L1 (1996); ibid 195, L261 (1999); L. Berger, Phys. Rev. B 54, 9353 (1996); J. Appl. Phys. 89, 5521 (2001).

[2] J. A. Katine, F. J. Albert, R. A. Buhrman, E. B. Myers, and D. C. Ralph, Phys. Rev. Lett. 84, 3149 (2000); F. J. Albert, J. A. Katine, R. A. Buhrman, and D. C. Ralph, Appl. Phys. Lett. 77, 3809 (2000); J. Grollier, V. Cros, A. Hamzic, J. M. George, H. Jaffrès, A. Fert, G. Faini, J. Ben Youssef, and H. Legall, Appl. Phys. Lett. 78, 3663
(2001).

[3] Peter M. Levy and Jianwei Zhang, to be published.

[4] M.D. Stiles and A. Zangwill, Phys. Rev. B 66, 014407 (2002)

[5] J.C. Slonczewski, J. Mag. Mag. Mater. 247, 324 (2002).

[6] H.E. Camblong, P.M. Levy and S. Zhang, Phys. Rev. B51, 16052 (1995).

[7] A. Brataas, Yu.V. Nazarov, and G.E.W. Bauer, Phys. Rev. Lett. 84, 2481 (2000) and D.H. Hernando, Y.V. Nazarov, A. Brataas, and G.E.W. Bauer, Phys. Rev.B 62, 5700 (2000); Alexey Kovalev, Arne Brataas and Gerrit E.W. Bauer, ibid 66, 224424 (2002); Gerrit.E.W. Bauer, Yaroslav Tserkovnyak, Daniel Huertas-Hernando and Arne Brataas, ibid 67, 094421 (2003).

[8] M.D. Stiles and A. Zangwill, J.Appl. Phys. 91, 6812 (2002).

[9] The off diagonal elements of a density matrix $\left|\phi_{a}\right\rangle<$ $\phi_{b} \mid$ refer to coherences between states; only the diagonal elements $a=b$ represent the occupancy of a state.

[10] see Transport Phenomena by H. Smith and H.H. Jensen; in particular Sec. 1.13.

[11] J. Rammer and H. Smith, Rev. Mod. Phys. 58, 323 (1986)

[12] T. Valet and A. Fert, Phys. Rev. B48, 7099 (1993).

[13] See Stiles Ref. 3 for an excellent discussion of how oscillating but non-decaying functions can interfere to produce a seemingly exponential decay.

[14] Jianwei Zhang, P.M. Levy and Shufeng Zhang, Bull. Amer. Phys. Soc. 48, 117 (2003); P.M. Levy, Jianwei Zhang and Vladimir P Antropov, ibid 48, 821 (2003).

[15] S. Zhang, P.M. Levy and A. Fert, Phys. Rev. Lett. 88, 236601 (2002).

[16] This identification of the magnetic part of the energy of a band state has been suggested by Professor Ingrid Mertig, privgate communication.

[17] The sharp peak in the distribution of $d_{J}(k)$ in the $\Gamma-X$ direction produces a long range tail in the transverse spin current whose amplitude is small compared to the main response coming form the average $\overline{d_{J}(k)}$. In the ballistic limit it does not decay; however it will when one considers diffusive scattering in the bulk of the layers.

[18] In Co for example there are three minority bands crossing the Fermi surface. We consider the only one deemed to make a significant contribution to electron transport.

[19] When the current is first turned on there is a discontinuity in the transverse component of the spin current as envisaged by others; however in steady state this is replaced by a more gradual rotation of the polarization. Therefore in steady state the spin torques and angular dependence of the resistance are different than those found from calculations which do not take account of these accumulations.

[20] The results of our self-consistent determination of transport across noncollinear multilayers will be presented in a forthcoming publication.

[21] A. Shpiro, P.M. Levy and S. Zhang, Phys. Rev.B 67,104430 (2003). 